\title{
MODELLING AND ANALYSIS OF NORMAL AND ATHEROSCLEROTIC BLOOD VESSEL MECHANICS USING 3D FINITE ELEMENT MODELS
}

\author{
K. Kamalanand ${ }^{1}$ and S. Srinivasan ${ }^{2}$ \\ Department of Instrumentation Engineering, Madras Institute of Technology, Anna University, Tamil Nadu, India \\ E-mail: ${ }^{1}$ kkamalanandmit@gmail.com, ${ }^{2}$ srini@ mitindia.edu
}

\begin{abstract}
In order to prevent, diagnose and treat vascular disease, detailed knowledge of blood vessel mechanics is essential. In this work, 3D finite element models of normal vessel and atherosclerotic vessel with $50 \%$ and $90 \%$ plaque deposition were developed. The developed models were meshed with tetrahedral elements using the Delaunay triangulation method and were simulated using actual blood pressure signals. Based on the transient analysis performed on the developed models, the parameters such as strain energy density and entropy per unit volume were obtained. Results demonstrate that an inverse relation exists between the considered mechanical parameters and the percentage of plaque deposited on the inner vessel wall. It was observed that the parameters decrease linearly with increasing plaque percentage. In this paper, the objectives of the study, the methodology and significant observations are discussed in detail.
\end{abstract}

Key words:

Blood Vessel Mechanics, Atherosclerosis, Plaque, Finite Element Model

\section{INTRODUCTION}

Atherosclerotic vascular disease is the most common cause of morbidity and mortality in developed countries, and the world-wide importance of acute vascular syndromes is increasing. As people age, they tend to develop fatty plaques within the walls of their blood vessels [1]. Blood vessels display highly nonlinear, elastic and anisotropic mechanical behavior and also exhibit complex material properties. The blood vessel mechanics change from vessel to vessel [2], change with ageing [3], and pathologies [4,5]. Hence, knowledge of blood vessel mechanics is fundamental to the understanding of vascular function in health and disease.

Even though a large amount of information is available on the histological structure of arteries [6], relatively little attention has been paid to the mechanical properties of the arteries. Measurements of this nature are in fact of more value to the physiologist than even detailed qualitative descriptions of the blood vessel [7]. However, experimental studies have several limitations including the time and expense of conducting these experiments and the difficulty in replicating in vivo conditions $[4,8]$.

Understanding the mechanical response of blood vessels to physiologic loads is necessary before ideal therapeutic solutions can be realized. For this reason, blood vessel constitutive models are needed [9]. In recent years, computational techniques such as finite element methods have been used increasingly by researchers seeking to understand vascular dynamics. These methods can augment the data provided by in vitro and in vivo methods by enabling a complete characterization of vessel mechanics. The analytic results can help physicians in the clinic, both in designing and in choosing appropriate therapies [1, 1012].

The finite element method is a powerful technique for finding approximate solution of a partial differential equation where the domain boundaries of a given problem are so complex that other approaches have difficulties or fail [13]. It has now become one of the fundamental numerical approaches for solving problems arising in many applications, including biomedical simulation. In the finite element method, a complex domain is discretized into a number of elements, such as that a set of basis functions can be defined on the elements to approximate the solution [1].

The objective of this work is to develop 3D Finite Element Models of normal and atherosclerotic blood vessels with various levels of plaque deposition and to investigate the influence of plaque deposition on the arterial vessel mechanics using parameters such as strain energy density and entropy per unit volume.

\section{METHODOLOGY}

\subsection{GENERATION OF 3D GEOMETRIC MODEL OF BLOOD VESSEL}

The 3D model of a section of the thoracic aorta was developed using Comsol 3.5a. FEM models were developed for normal vessel, vessel with $50 \%$ plaque deposition and vessel with $90 \%$ plaque deposition and are shown in Fig.1(a), (b) and (c) respectively.

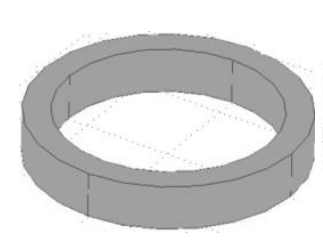

(a) (b)

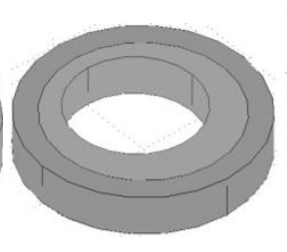

(c)

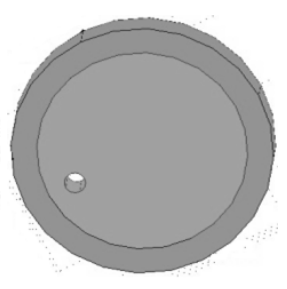

Fig.1 (a) normal blood vessel geometry, (b) atherosclerotic vessel with $50 \%$ plaque deposition and (c) atherosclerotic vessel with $90 \%$ plaque deposition

The geometry and mechanical properties of the vessel were adopted from literature [6]. The stiffness of the plaque component was taken to be 0.5 times that of the vessel stiffness. Further, the boundary conditions were applied to the developed models and the developed volumes were meshed with tetrahedral elements using the Delaunay triangulation method. The mesh quality was improved by fine tuning the mesh. 


\subsection{BASIS FUNCTION}

The stress-strain relationship is developed for a general strain energy function based on strain invariants. The first and second strain invariants $\overline{I_{1}}, \overline{I_{2}}$ and volume ratio $\mathrm{J}$ are chosen as the variables in the strain energy function [14]. They are defined as:

$$
\begin{gathered}
\bar{I}_{1}=\bar{\lambda}_{1}^{2}+\bar{\lambda}_{2}^{2}+\bar{\lambda}_{3}^{2} \\
\bar{I}_{1}={\overline{\lambda_{1}}}^{2}{\overline{\lambda_{2}}}^{2}+\bar{\lambda}_{2}^{2} \bar{\lambda}_{3}^{2}+\bar{\lambda}_{1}^{2} \bar{\lambda}_{3}^{2} \\
\bar{\lambda}_{k}=J^{-\frac{1}{3}} \lambda_{k}, \quad k=1,2,3
\end{gathered}
$$

where, $\lambda_{k}(k=1,2,3)$ are the principal stretch ratios and $\mathbf{J}$ is the total volume ratio. The strain energy function can be expressed in terms of $\overline{I_{1}}, \overline{I_{2}}$ and $\mathrm{J}$,

$$
U=u\left(\bar{I}_{1}, \bar{I}_{2}, J\right)
$$

Further, the stress-strain behavior is defined using the following equations. Writing the current position of a material point as $x$ and the reference position of the same point as $X$, the deformation gradient is then defined as

$$
F=\frac{\partial x}{\partial X}=\frac{\partial x_{i}}{\partial X_{j}} \quad(i, j=1,2,3)
$$

Then $J$, the total volume change at the point, is

$$
J=\operatorname{det}(F)
$$

For simplicity, the deformation gradient with the volume change eliminated is defined as

$$
\bar{F}=J^{-\frac{1}{3}} F
$$

Then, the deviatoric stretch matrix is introduced as

$$
\bar{B}=\bar{F} \cdot \bar{F}^{T}
$$

So that the first strain invariant is given by

$$
\overline{I_{1}}=\operatorname{trace} \bar{B}=\bar{B}_{i i} \quad(i=1,2,3)
$$

and the second strain invariant is given by

$$
\overline{I_{2}}=\frac{1}{2}\left\{(\operatorname{trace}(\bar{B}))^{2}-\operatorname{trace}(\bar{B} \cdot \bar{B})\right\}
$$

Then the stresses associated with the strain energy function are given by

$$
\sigma=\frac{2}{J} \operatorname{dev}\left\{\left(\frac{\partial U}{\partial \bar{I}_{1}}+\overline{I_{1}} \frac{\partial U}{\partial \bar{I}_{2}}\right) \bar{B}-\frac{\partial U}{\partial \bar{I}_{2}} \bar{B} \cdot \bar{B}\right\}+\frac{\partial U}{\partial J} I
$$

where, $\operatorname{dev}$ means deviatoric and is calculated as $\operatorname{dev}(\mathrm{A})=\mathrm{A}-$ $1 / 3$ trace(A) for matrix $A$. I is the Identity matrix. For incompressible material, $\mathrm{J}=1, u$ is a function of $\mathrm{I}_{1}$ and $\mathrm{I}_{2}$ only [14]. The potential function of the arterial material is as follows:

$$
\bar{U}=\frac{\mu_{1}}{2}\left(\overline{I_{1}}-3\right)+\frac{K_{1}}{2}(J-1)^{2}
$$

The material constants are the shear modulus, $\mu_{l}$ and the bulk modulus, $K_{l}$.

\subsection{FORCING FUNCTION}

The actual blood pressure (BP) signals were obtained from the MIT database of clinical signals (www.physiobank.net). The BP signal was modelled as a function of time using the sum of sinusoids model of the following form:

$$
y(t)=a_{1} \sin \left(b_{1} t+c_{1}\right)+a_{2} \sin \left(b_{2} t+c_{2}\right)+\ldots . .+a_{n} \sin \left(b_{n} t+c_{n}\right)
$$

where, $a_{1}, a_{2}, \ldots . a_{n}, b_{1}, b_{2}, \ldots, b_{n}, c_{1}, c_{2}, \ldots c_{n}$ are parameters of the model, ' $t$ ' is the time and, ' $y$ ' refers to the amplitude of the BP signal.

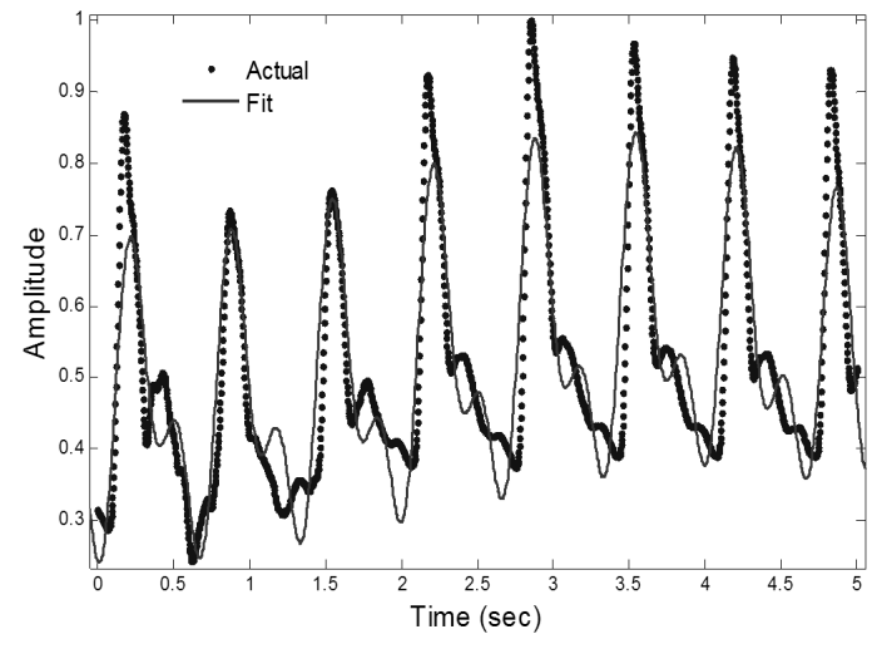

Fig.2. The actual BP signal and the computed fit

Several models were developed by increasing the number of model terms. A model with the five sine terms was found to have the least error. The actual BP signal and the fitting function are shown in Fig.2. The obtained function was used as the forcing function for simulating the developed FEM models of normal and abnormal blood vessels. Based on the simulations performed, the parameters such as total displacement, strain energy density and entropy per unit volume were obtained.

\section{RESULTS AND DISCUSSION}

The variation of strain energy density of the normal vessel, vessel with $50 \%$ plaque formation and vessel with $90 \%$ plaque formation is shown as a function of time in Fig.3(a), (b) and (c) respectively. The variation of strain energy density in the case of normal vessel is found to be periodic. However, in the case of atherosclerotic vessels, the variation in strain energy density appears to be non-periodic.

Fig.4(a), (b) and (c) shows the variation of entropy per unit volume in the normal vessel, vessel with $50 \%$ plaque formation and vessel with $90 \%$ plaque formation as a function of time. The variation of entropy per unit volume in normal and atherosclerotic vessel models is found to be similar to the variation in strain energy density.

The variation of strain energy density is shown as a function of the percentage of plaque deposited on the inner wall of the vessel, in Fig.5. It is seen that the strain energy density decreases linearly with increasing plaque percentage. Further, it appears that the considered parameters are useful for analyzing the effect of physiological loads in normal and atherosclerotic blood vessels. 


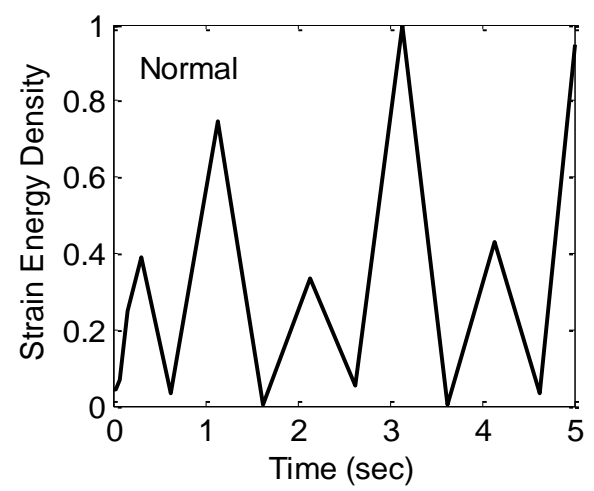

(a)

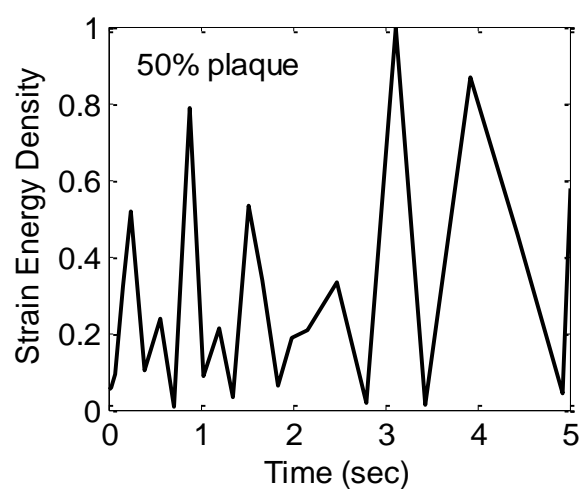

(b)

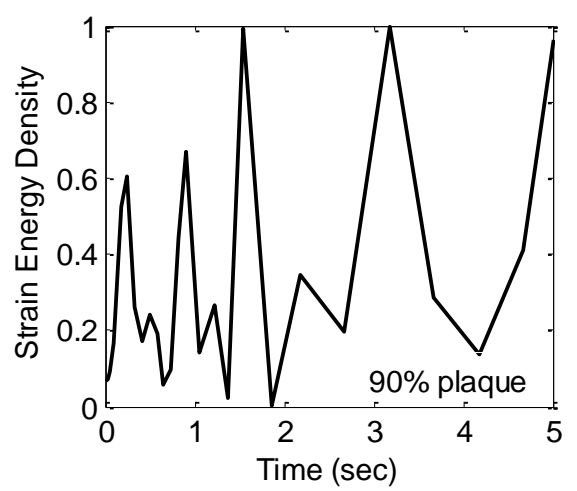

(c)

Fig.3. The variation of strain energy density in the (a) normal vessel, (b) vessel with $50 \%$ plaque formation and (c) vessel with $90 \%$ plaque formation shown as a function of time

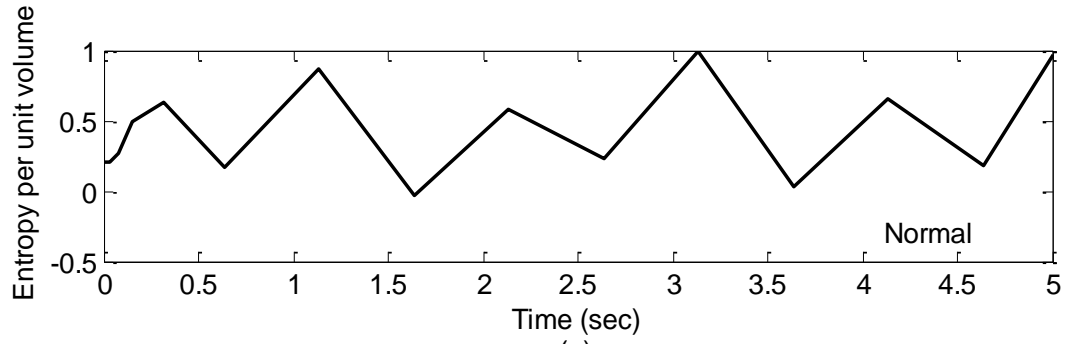

(a)

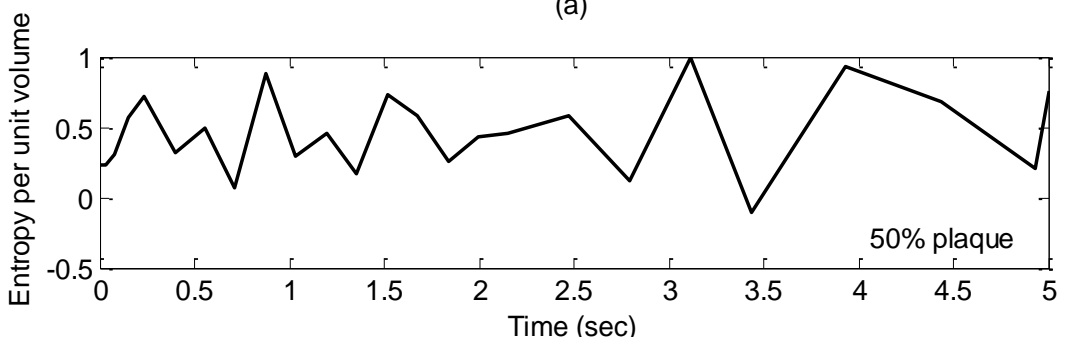

(b)

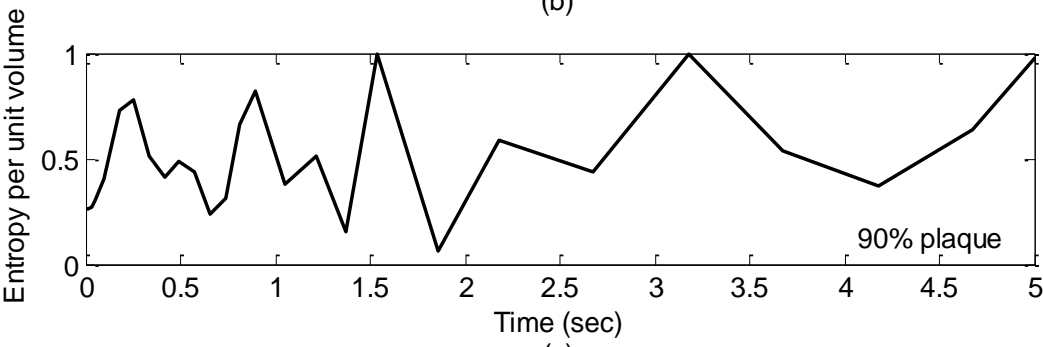

(c)

Fig.4. The variation of entropy per unit volume in the (a) normal vessel, (b) vessel with 50\% plaque formation and (c) vessel with $90 \%$ plaque formation shown as a function of time 


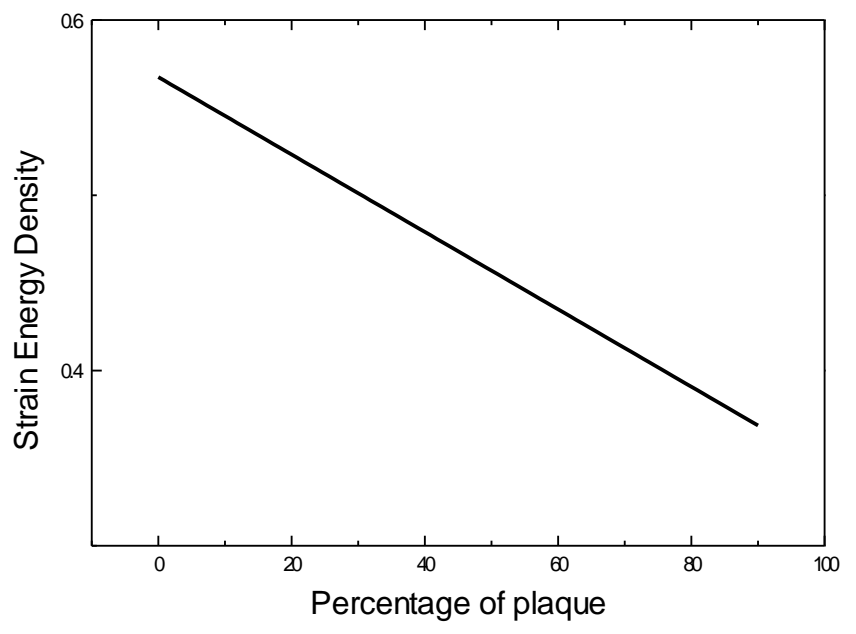

Fig.5. Variation of strain energy density shown as a function of the percentage of plaque deposition

\section{CONCLUSION}

In this work, 3D finite element models of normal vessel and atherosclerotic vessels with $50 \%$ and $90 \%$ plaque deposition were developed using COMSOL 3.5a. Fixed boundary conditions were applied to the outer diameter considering the effect of surrounding tissues and structures. The inner diameter was set to be free and the developed models were simulated using a physiological blood pressure signal. Further, tetrahedral elements were used for meshing the developed models. The tetrahedral elements were obtained using an extension of the Delaunay triangulation method. The transient analysis was performed on the developed models the parameters such as strain energy density and entropy per unit volume were analyzed for normal and atherosclerotic vessels.

Results demonstrate that the variation of parameters such as strain energy density and entropy per unit volume in the case of normal vessel appears to be periodic and in the case of atherosclerotic vessels, the variation in these parameters appears to be non-periodic. It appears that an inverse relation exists between the considered mechanical parameters and the percentage of plaque deposited on the inner vessel wall. It was further observed that the parameters decrease linearly with increasing plaque percentage. This study seems to be clinically important since the analysis of vascular mechanics in normal and diseased states is essential for designing stents, surgery planning, treatment and diagnosis of vascular diseases.

\section{REFERENCES}

[1] C.F. Carmen, A. Stelian, C.M. Cătălina, I. Luminita and C.D. Aurora, "Finite Element Analysis For A Simplified Model Of A Blood Vessel With Lesion", Annals Of The Oradea University, Fascicle of Management and Technological Engineering, Vol. IX, No. XIX, NR1.
[2] R.H. Cox, "Passive mechanics and connective tissue composition of canine arteries", American Journal of Physiology, Vol. 234, No. 5, pp. H533-H541, 1978.

[3] L. Andreotti et al., "Aortic connective tissue in ageing-a biochemical study", Angiology, Vol. 36, No. 12, pp. 872 879, 1985.

[4] M.A. Zulliger, P. Fridez, K. Hayashi and N. Stergiopulos, "A strain energy function for arteries accounting for wall composition and structure", Journal of Biomechanics, Vol. 37, No. 7, pp. 989-1000, 2004.

[5] F.L. Wuyts et al., "Elastic properties of human aortas in relation to age and atherosclerosis: a structural model", Physics in Medicine \& Biology, Vol. 40, No. 10, pp. 15771597, 1995.

[6] A. Hager et al., "Diameters of the thoracic aorta throughout life as measured with helical computed tomography", $J$ Thorac Cardiovasc Surg, Vol. 123, No. 6, pp.1060-1066, 2002.

[7] B.M. Learoyd and M.G. Taylor, "Alterations with Age in the Viscoelastic Properties of Human Arterial Walls", Circulation Research, Vol. 18, No. 3, pp.278-292, 1966.

[8] A.J.M Spencer, “Continuum Mechanics, Longman Scientific \& Technical", Essex, 1980.

[9] R.P. Vito and S.A. Dixon, "Blood Vessel Constitutive models,-1995-2002", Annual. Reviews of Biomedical Engineering, Vol. 5, pp.413-439, 2003..

[10] S.J. Payne, "A Two-Layer model of the Static Behaviour of Blood Vessel Walls", Proceedings of the International conference IEEE Engineering in Medicine and Biology Society, Vol. 5, No. 11, pp. 3692-3695, 2004.

[11] Ed VanBavel, P. Siersma and Jos A.E. Spaan, "Elasticity of passive blood vessels: a new concept", American Journal of Physiology Heart Circulatory Physiology, Vol. 285, No. 5, pp. H1986-H2000, 2003.

[12] M. Orosz et al., "Validity of viscoelastic models of blood vessel wall”, Acta Physiologica Hungarica, Vol. 86, No. 3 4, pp.265-271, 1999.

[13] Z. Yu, M.J. Holst and J.A. McCammon, "High-fidelity geometric modeling for biomedical applications", Finite Elements in Analysis and Design, Vol. 44, No. 11, pp. 715723, 2008.

[14] J.R. Shewchuk, "Lecture notes on Delaunay mesh generation", http://citeseer.ist.psu.edu/shewchuk99lecture.html.

[15] Y. Shen, K. Chandrashekhara, W.F. Breig and L.R. Oliver, "Finite element analysis of V-ribbed belts using neural network based hyperelastic material model", International Journal of Non-Linear Mechanics, Vol. 40, No. 6, pp.875890,2005 